\title{
II. The effect of a surface-film in total reflexion
}

\section{Robert A. Houstoun M.A. B.Sc.}

To cite this article: Robert A. Houstoun M.A. B.Sc. (1905) II. The effect of a surface-film in total reflexion , Philosophical Magazine Series 6, 10:55, 12-24, DOI: 10.1080/14786440509463339

To link to this article: http://dx.doi.org/10.1080/14786440509463339

$$
\text { 册 Published online: } 16 \text { Apr } 2009 .
$$

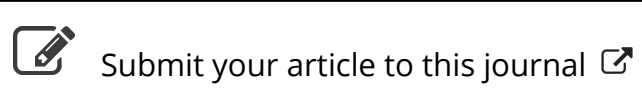

\footnotetext{
Џll Article views: 2
}

Q View related articles $\square$

4 Citing articles: 1 View citing articles 4 
which, in going through, forms the osmotic current *. On this point the work of Nernst $\dagger$ and Flusin $\ddagger$ may be referred to. During the last year the writer has been working on the osmotic pressure of ethyl-alcohol solutions with different membranes. One experiment may be mentioned here (it is not put forward as original). Pure alcohol and pure water were separated by a gutta-percha tissue, the water being within the cell. A large pressure was set up, sbowing an inflow from the alcohol to the water. With a copperferrocyanide membrane the direction is reversed. The cause of the current is the same in each case, namely, the mutual potential energy of solution of the liquids; the direction of the current is conditioned by the membrane.

The experimental work of this paper was carried out at the Cavendish I Laboratory. I wish to take this opportunity of thanking Prof. J. J. Thomson for his kindness during the progress of the work.

II. The Effect of a Surface-Film in Total Reflexion. By Robert A. Houstoun, M.A., B.Sc., Glasgow University 1851 Exhibition Scholar $\$ \|$.

T'T is well known that Fresnel's Laws for determining the phase and amplitude of a reflected or refracted light-wave from those of the incident wave have not been found to give the exact experimental result, when investigated with the most accurate experimental means. There is a difference between the calculated and observed results, greater than the experimental error. It is assumed in deriving Fresnel's laws, that at the surface where reflexion takes place the index of refraction changes discontinuously in going from the one medium to the other. The deviation from Fresnel's laws has been satisfactorily explained on the assumption that there is a "surface-film" between the two media. This surface-film can be regarded as a transition-layer; in which the index of refraction changes continuously from the value it has in the

* Since this paper was written, a similar view has been arrived at by Prof. Kahlenburg (Phil. Mag. Feb. 1905, p. 228) on similar lines of work.

$\dagger$ Zeit. Phys. Chem. vi. p. 37 (1890).

$\ddagger$ Comptes Rendus, exxxi. p. 1308 (Dee. 31, 1900).

\$ Communicated by Professir A. Gray, F.R.S.

II "Wirkuug einer Oberflächenschicht bei Total-reflexion," R. A. Houstoun, Göttinger Nachrichten, 1903, p. 352. "Ueber die Wirkun'. einer Oberflächenschicht bei Total-reflexion." R. A. Houstoun, Phys. Zeitsch, ift, vi. p. 208. 
one medium to the value it has in the other; or it can be regarded as a nearly homogeneous layer with a definite index of refraction, produced in polishing the surface. 'The formula for the deviation has been proved experimentally by Jamin * and Quincke $\dagger$ for the case of ordinary reflexion. 'Ihis paper describes some observations made to prove the formula in the case of total reflexion, which was first given by Drude $\ddagger$, though it follows naturally from the formula for the case of ordinary reflexion, which was found some years earlier. Fresnel's laws for the case of total reflexion were proved by Jamin $\S$ and Quincke $\|$, who worked with fresh surfaces for which the effect of the surface-film would be naturally very small.

The apparatus used was a spectroscope, the collimator of which was fitted with a polarizing vicol, and the telescope of which was fitted with a Soleil-Babinet compensator and an analysing nicol. A sodium-flame was the source of light. The incident light was polarized at $45^{\circ}$ to the vertical ; after total reflexion it was once more made plane-polarized by the compensator, and then cut off by the crossed analyser.

The Soleil-Babinet compensator consists essentially of a quartz plate and two quartz wedges with equal angles. The

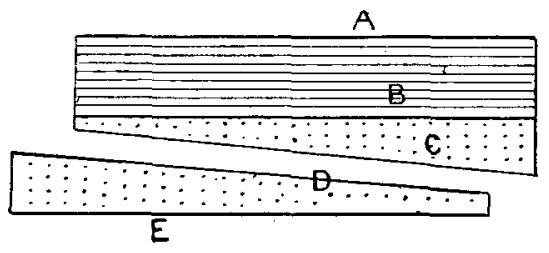

two wedges have their optical axes in the same direction, parallel or perpendicular to the edge of the wedge; the wedges are parallel, but in opposite directions. The axis of the plate is perpendicular to the axes of the wedges. The relative phase-difference produced by reflexion \&c. is compensated by moving the one quartz wedge relatively to the other wedge and the plate, which are fixed together.

The elementary theory disregards the reflected waves. It assumes also that the axes of the two wedges and the plate are

* Jamin, Ann. de Phys. et Chim. (3) vol. xxix. p. 263 (1850).

+ Quincke, Poggr. Ann. vol. exxviii. p, 355 (1866).

† P. Drude, Wied. Ann. vol. xliii. p. 146 (1891).

\$ J. Jamin, Ann. de Phys. et Chim. (3) vol. xxx. p. 257 (1850).

|| G. Quincke, Pogg. Ann. vol. cxxvii. p. 217 (1866). 
set exactly. This second cause of error was investigated theoretically, and does not seem to be an appreciable cause of error in the observations made. The first cause of error, the effect of inner reflexion, has been discussed by Prof. W. Voigt* In the instrument used, the rays reflected on the surfaces $\mathrm{C}$ and $D$ form separate images in the field of vision, and the intensity of the light reflected at $B$ and again at $A$ is too small to be taken into consideration. There remains only the light reflected on the surfaces $\mathrm{E}$ and $\mathrm{A}$, the intensity of which is perhaps $\frac{1}{700}$ of the intensity of the light that comes direct through. As the distance between the surfaces $\mathrm{A}$ and $\mathrm{E}$ is great, and the light is not absolutely homogeneous, this error does not come into consideration.

In the elementary theory it is assumed that light is propagated in quartz in two waves, plane-polarized at right angles to one another. Prof. Voigt has, however, shown in a recent article $t$, that it is propagated in two elliptically polarized vibrations, the ellipses being long and thin, having their long axes perpendicular to one another, and being traversed in opposite directions. The effect of the ellipticity of the vibrations on observations made with the compensator was investigated, and a result in accordance with Prof. Voigt's theory obtained. The investigation is given here.

For simplicity we can consider the compensator as consisting of two plane parallel plates of quartz, with their axes $\mathrm{H}_{1}$ and $\mathrm{H}_{2}$ exactly at right angles to one another. Let us

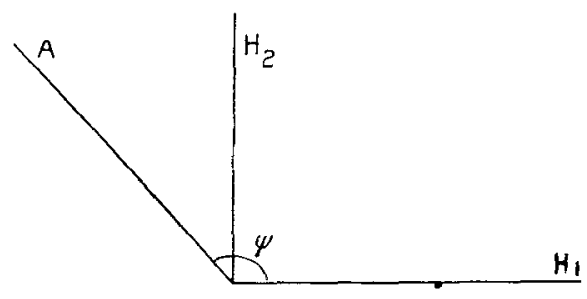

suppose that the incident light consists of two vibrations, $\mathrm{E} \sin T$ along $\mathrm{H}_{1}$, and $\mathrm{E}^{\prime} \sin \mathrm{T}^{\prime}$ along $\mathrm{H}_{2}$. To take account of the elliptic propagation in the quartz we must replace these two linear vibrations by four elliptic vibrations, a righthanded and a left-handed vibration having their long axes in

* W Voigt, Wied. Ann. vol. xxii. p. 236 (1884).

$\dagger$ W. Voigt, Gött. Nach. 1903, p. $\mathbf{1} 55$. 
each of the directions $\mathrm{H}_{1}$ and $\mathrm{H}_{2} ; i$. e. we must write

$\mathrm{E}\left(\left\{1-\kappa^{2}\right\} \sin \mathrm{T}+\kappa^{2} \sin \mathrm{T}\right)+\mathrm{E}^{\prime}\left(\kappa \cos \mathrm{T}^{\prime}-\kappa \cos \mathrm{T}^{\prime}\right)$ in the direction $\mathrm{H}_{1}$, and

$\mathrm{E}(\kappa \cos \mathrm{T}-\kappa \cos \mathrm{T})+\mathrm{E}^{\prime}\left(\left(1-\kappa^{2}\right) \sin \mathrm{T}^{\prime}+\kappa^{2} \sin \mathrm{T}^{\prime}\right)$ in the direction $\mathrm{H}_{2}$.

$\boldsymbol{\kappa}$ is very small. The underlined terms are right-handed or negative ellipses. Let the right-handed vibrations receive the phase-retardation $r_{1}$ in passing through the first plate, and the left-handed vibrations the phase-retardation $l_{1}$. Then, if we disregard the $\kappa^{2}$ terms, after the first plate the two components are

$$
\mathrm{E} \sin \left(\mathrm{T}-r_{\mathrm{i}}\right)+\mathrm{E}^{\prime}\left(\kappa \cos \left(\mathrm{T}^{\prime}-l_{1}\right)-\kappa \cos \left(\mathrm{T}^{\prime}-r_{1}\right)\right)
$$

and

$$
\mathrm{E}\left(\kappa \cos \left(\mathrm{T}-r_{1}\right)-\kappa \cos \left(\mathrm{T}^{\prime}-l_{1}\right)\right)+\mathrm{E}^{\prime} \sin \left(\mathrm{T}^{\prime}-l_{1}\right) .
$$

These may be written

$$
\left(\mathrm{E}^{2}+\mathrm{E}^{\prime 2} \kappa^{2}\right)^{\frac{1}{2}} \sin \left(\mathrm{T}-r_{1}-\tan ^{-1} \frac{\kappa \mathrm{E}^{\prime}}{\mathrm{E}}\right)+\mathrm{E}^{\prime} \kappa \cos \left(\mathrm{T}^{\prime}-l_{1}\right),
$$

and

$$
\left(\mathrm{E}^{\prime 2}+\mathrm{E}^{2} \kappa^{2}\right)^{\frac{1}{2}} \sin \left(\mathrm{T}^{\prime}-l_{1}-\tan ^{-1} \frac{\kappa \mathrm{E}}{\mathrm{E}^{\prime}}\right)+\mathrm{E} \kappa \cos \left(\mathrm{T}-r_{1}\right) .
$$

Let $r_{2}$ and $l_{2}$ be the corresponding phase-retardations produced by the second plate. Then, if we consider only that portion of the phase-retardation wlich varies with the thickness of the plate, we shall have, after the second plate, in each of the directions $\mathrm{H}_{1}$ and $\mathrm{H}_{2}$, four vibrations with the phaseretardations

$$
r_{1}+r_{2}, \quad r_{1}+l_{2}, \quad l_{1}+r_{2}, \quad \text { and } l_{1}+l_{2} .
$$

Hence we shall have vibrations with those phase-retardations in the principal plane of the analysing nicol. In the expression for the resultant intensity there will occur a number of terms, of the form

$\mathrm{A} \cos \left\{\left(r_{1}+r_{2}\right)-\left(l_{1}+l_{2}\right)+a\right\}+\mathrm{B} \cos \left\{\left(r_{1}+r_{2}\right)-\left(r_{1}+l_{2}\right)+b\right\}$

$$
\begin{aligned}
& +\mathrm{C} \cos \left\{\left(r_{1}+r_{2}\right)-\left(l_{1}+r_{2}\right)+e\right\}+\mathrm{D} \cos \left\{\left(r_{1}+l_{2}\right)-\left(l_{1}+r_{2}\right)+d\right\} \\
& +\mathrm{E} \cos \left\{\left(r_{1}+l_{2}\right)-\left(l_{1}+l_{2}\right)+e\right\}+\mathrm{F} \cos \left\{\left(l_{1}+r_{2}\right)-\left(l_{1}+l_{2}\right)+f\right\} .
\end{aligned}
$$

Write $r_{1}-l_{1}=\delta_{1}$ and $r_{2}-l_{2}=-\delta_{2}$. Then we may write the six phases

$$
\delta_{1}-\delta_{2}+a,-\delta_{2}+b, \delta_{1}+c, \delta_{1}+\delta_{2}+d, \delta_{1}+e, \text { and }-\delta_{2}+f \text {. }
$$


As the one wedge is moved past the other, $\delta_{2}$ changes ; and each of the six terms gives a similar system of maxima and minima. It is the system with the phase $\delta_{1}-\delta_{2}+a$ on which the working of the instrument depends. Its intensity is $\frac{1}{\kappa}$ times the intensity of the others; and the position of its minima is not appreciably affected by the other systems.

Hence we can disregard the other systems, $i$. e., we can disregard the second terms in the expressions (I.) and (II.). On entering the second plate the first terms become

$$
\begin{aligned}
& \left(\mathrm{E}^{2}+\mathrm{E}^{\prime 2} \kappa^{2}\right)^{\frac{1}{3}}\left\{\left(1-\kappa^{2}\right) \sin \left(\mathrm{T}-r_{1}-\tan ^{-1} \frac{\kappa \mathrm{E}^{\prime}}{\mathrm{E}}\right)+\kappa^{2} \sin \left(\mathrm{T}-r_{1}-\tan ^{-1} \frac{\kappa \mathrm{E}^{\prime}}{\mathrm{E}}\right)\right\} \\
& +\left(\mathrm{E}^{\prime 2}+\mathrm{E}^{2} \kappa^{2}\right)^{\frac{1}{2}}\left\{\kappa \cos \left(\mathrm{T}^{\prime}-l_{1}-\tan ^{-1} \frac{\kappa \mathrm{E}}{\mathrm{E}^{\prime}}\right)-\kappa \cos \left(\mathrm{T}^{\prime}-l_{1}-\tan ^{-1} \frac{\kappa \mathrm{E}}{\mathrm{E}^{\prime}}\right)\right\} \\
& \quad \text { and } \\
& \left(\mathrm{E}^{2}+\mathrm{E}^{\prime \prime} \kappa^{2}\right)^{\frac{1}{2}}\left\{\frac{\left.\kappa \cos \left(\mathrm{T}-r_{1}-\tan ^{-1} \frac{\kappa \mathrm{E}^{\prime}}{\mathrm{E}}\right)-\kappa \cos \left(\mathrm{T}-r_{1}-\tan ^{-1} \frac{\kappa \mathrm{E}^{\prime}}{\mathrm{E}}\right)\right\}}{+\left(\mathrm{E}^{\prime 2}+\mathrm{E}^{2} \kappa^{2}\right)^{\frac{1}{2}}\left\{\left(1-\kappa^{2}\right) \sin \left(\mathrm{T}^{\prime}-l_{1}-\tan ^{-1} \frac{\kappa \mathrm{E}}{\mathrm{E}^{\prime}}\right)+\kappa^{2} \sin \left(\mathrm{T}^{\prime}-l_{1}-\tan ^{-1} \frac{\kappa \mathrm{E}}{\mathrm{E}^{\prime}}\right)\right\}}\right.
\end{aligned}
$$

If we disregard the $\kappa^{2}$ terms, on leaving the second plate the two components are

$$
\begin{aligned}
&\left(\mathrm{E}^{2}+\mathrm{E}^{\prime 2} \kappa^{2}\right)^{\frac{1}{2}} \sin \left(\mathrm{T}-r_{1}-r_{2}-\tan ^{-1} \frac{\kappa \mathrm{E}^{\prime}}{\mathrm{E}}\right)+\left(\mathrm{E}^{\prime 2}+\mathrm{E}^{2} \kappa^{2}\right)^{\frac{1}{2}} \\
&\left\{\kappa \cos \left(\mathrm{T}^{\prime}-l_{1}-l_{2}-\tan ^{-1} \frac{\kappa \mathrm{E}}{\mathrm{E}^{\prime}}\right)-\kappa \cos \left(\mathrm{T}^{\prime}-l_{1}-r_{2}-\tan ^{-1} \frac{\kappa \mathrm{E}}{\mathrm{E}^{\prime}}\right)\right\}
\end{aligned}
$$

and

$$
\begin{array}{r}
\left(\mathrm{E}^{2}+\mathrm{E}^{\prime 2} \kappa^{2}\right)^{\frac{1}{2}}\left\{\kappa \cos \left(\mathrm{T}-r_{1}-r_{2}-\tan ^{-1} \frac{\kappa \mathrm{E}^{\prime}}{\mathrm{E}}\right)-\kappa \cos \left(\mathrm{T}-r_{1}-l_{2}-\tan ^{-1} \frac{\kappa \mathrm{E}^{\prime}}{\mathrm{E}^{-}}\right)\right\} \\
+\left(\mathrm{E}^{\prime 2}+\mathrm{E}^{2} \kappa^{2}\right)^{\frac{1}{2}} \sin \left(\mathrm{T}^{\prime}-l_{1}-l_{2}-\tan ^{-1} \frac{\kappa \mathrm{E}}{\mathrm{E}^{\prime}}\right) .
\end{array}
$$

We can disregard the third term in the first component and the second term in the second component for the same reason that the second terms were disregarded above. If we multiply the first component by $\cos \psi$ and the second by $\sin \psi$ and add, we get the vibration in the principal plane of the analysing nicol

$$
\begin{aligned}
& \left\{\left(\mathrm{E}^{2}+\mathrm{E}^{\prime 2} \kappa^{2}\right) \cos ^{2} \psi+\mathrm{E}^{2} \kappa^{2} \sin ^{2} \psi\right\}^{\frac{1}{2}} \sin \left(\mathrm{T}-r_{1}-r_{2}-\tan ^{-1} \frac{\kappa \mathrm{E}^{\prime}}{\mathrm{E}}+\tan ^{-1} \kappa \tan \psi\right) \\
& +\left\{\left(\mathrm{E}^{\prime 2}+\mathrm{E}^{2} \kappa^{2}\right) \sin ^{2} \psi+\mathrm{E}^{\prime 2} \kappa^{2} \cos ^{2} \psi\right\}^{\frac{1}{2}} \sin \left(\mathrm{T}^{\prime}-l_{1}-l_{2}-\tan ^{-1} \frac{\kappa \mathrm{E}}{\mathrm{E}^{\prime}}+\tan ^{-1} \kappa \cot \psi\right)
\end{aligned}
$$


The resultant intensity is therefore

$$
\begin{aligned}
& \left(\mathrm{E}^{2}+2 \mathrm{E}^{\prime 2} \kappa^{2}\right) \cos ^{2} \psi+\left(\mathrm{E}^{\prime 2}+2 \mathrm{E}^{2} \kappa^{2}\right) \sin ^{2} \psi \\
& \left.+2\left\{\left(\mathrm{E}^{2}+\mathrm{E}^{\prime 2} \kappa^{2}\right) \cos ^{2} \psi+\mathrm{E}^{2} \kappa^{2} \sin ^{2} \psi\right\}^{\frac{2}{2}}\left\{\mathrm{E}^{\prime 2}+\mathrm{E}^{2} \kappa^{2}\right) \sin ^{2} \psi+\mathrm{E}^{\prime 2} \kappa^{2} \cos ^{2} \psi\right\} \\
& \quad \times \cos \left\{\mathrm{T}-\mathrm{T}^{\prime}-\left(r_{1}+r_{2}-l_{1}-l_{2}\right)-\tan ^{-1} \frac{\kappa \mathrm{E}^{\prime}}{\mathrm{E}}+\tan ^{-1} \kappa \mathrm{E}\right. \\
& +\mathrm{E}^{\prime} \\
& \left.+\tan ^{-1} \kappa \tan \psi-\tan ^{-1} \kappa \cot \psi\right\} .
\end{aligned}
$$

The extinction position of the compensator is therefore given by

$$
\begin{aligned}
\mathrm{T}-\mathrm{T}^{\mathrm{Y}}-\left(r_{1}+r_{2}-l_{1}-l_{2}\right) & -\tan ^{-1} \frac{\kappa \mathrm{E}^{\prime}}{\mathrm{E}}+\tan ^{-1} \frac{\kappa \mathrm{E}}{\mathrm{E}^{\prime}} \\
& +\tan ^{-1} \kappa \tan \psi-\tan ^{-1} \kappa \cot \psi=0 .
\end{aligned}
$$

It deviates from the position which we should obtain if the elliptic propagation of light in quartz were disregarded, by

$$
-\tan ^{-1} \frac{\kappa \mathrm{E}^{\prime}}{\mathrm{E}}+\tan ^{-1} \frac{\kappa \mathrm{E}}{\mathrm{E}^{\prime}}+\tan ^{-1} \kappa \tan \psi-\tan ^{-1} \kappa \cot \psi,
$$

or $\quad-2 \tan ^{-1} 2 \kappa \cot 2 \psi$,

since for the extinction position $\tan \psi \fallingdotseq \frac{\mathrm{E}}{\mathrm{E}^{\prime}}$. If $\mathrm{E}=\mathrm{E}^{\prime}$, as is the case with observations on total reflexion, then the deviation is zero; and this cause does not affect the aceuracy of the results.

Observations were made on this deviation. The zero of the compensator was determined for different positions of the crossed nicols, $i . e$. for different values of $\psi$. $\psi$ and $\kappa$ were determined from the observations. $\kappa$ was found to be 0.0016

\begin{tabular}{|c|c|c|c|c|c|c|c|}
\hline$\psi$ & $\begin{array}{l}\text { Observed } \\
\text { Deviation. }\end{array}$ & $\begin{array}{l}\text { Mean } \\
\text { Error }\end{array}$ & $\begin{array}{l}\text { Theoretical } \\
\text { Deviation. }\end{array}$ & $\psi$ & $\begin{array}{l}\text { Observed } \\
\text { Deviation. }\end{array}$ & $\begin{array}{l}\text { Mean } \\
\text { Error. }\end{array}$ & $\begin{array}{l}\text { Theoretical } \\
\text { Deviation. }\end{array}$ \\
\hline $88^{\circ}$ & 8.7 & $\stackrel{i}{1} \cdot 7$ & $\stackrel{8}{5} \cdot 2$ & $5 \stackrel{\circ}{50}$ & $-10 \div 2$ & $0 \cdot 2$ & $0^{\circ} \cdot 1$ \\
\hline $86 \ldots$ & 28 & 0.4 & 26 & 40 & $=0.0$ & 0.0 & $\begin{array}{l}0.1 \\
0.0\end{array}$ \\
\hline $83 \ldots$ & $1 \cdot 2$ & 0.4 & 1.4 & 25. & -0.4 & 0.2 & -0.3 \\
\hline 78 & $1 \cdot 0$ & $0 \cdot 2$ & 0.8 & 15. & $-0 \cdot \overline{6}$ & 0.0 & $-0 \cdot 7$ \\
\hline 75 & $0 \cdot 9$ & $0 \cdot 2$ & 0.6 & 5 & -28 & $0 \cdot 2$ & $-2 \cdot 0$ \\
\hline $65 \ldots$ & 0.0 & $0 \cdot 2$ & $0 \cdot 3$ & 2 & -54 & 0.8 & $-5 \cdot 6$ \\
\hline
\end{tabular}
as against values ranging from 0.0019 to 0.0012 given by Voigt in the above-mentioned article. $\kappa$ was then substituted in the formula, and the theoretical value of the deviation calculated. $\kappa$ is the ratio of the axes of the ellipse in which the light vibrates. It is very difficult to take readings for values of $\psi$ near $0^{\circ}$ or $90^{\circ}$

Plill. Mag. S. 6. Vol. 10. No. 55. July 1905. 
The first observations were made on a very old prism, the surface-films of which must have grown through age. The incident light was polarized at an angle of $45^{\circ}$ to the plane of incidence.

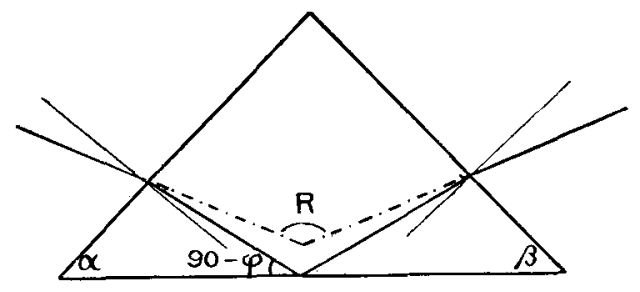

$\phi$, the angle of incidence, was calculated from $R$, the angle between the directions of the telescope and collimator, and $n$ the index of refraction, by the following formula :

$$
\mathrm{R}=\alpha+\beta+\sin ^{-1}(n \sin \{\phi-\alpha\})+\sin ^{-1}(n \sin \{\phi-\beta\}) .
$$

Observations were made for a number of angles, starting from the limiting angle of total reflexion. After some unsuccessful attempts, two good series of observations were obtained, - ten different angles and ten observations to each angle. Before the experiment the prism was left all night in alcohol, then washed thoroughly with water and dried with a linen cloth. It was placed in such a position upon the spectrometertable, that when it was rotated the light always fell upon the same part of the hypotenuse surface during the same series of observations. The two series of observations given were made for light that was totally reflected on different parts of the surface.

$\Delta$ is the observed phase-difference. In the next column to $\Delta$ stands the mean error to give an idea of the accuracy of the observations. $\quad \Delta_{0}$ is the phase-difference calculated aceording to Fresnel's formula.

Fresnel's formula is

$$
\tan \frac{1}{2} \Delta_{0}=-\sqrt{\frac{\sin ^{2} \phi-\frac{1}{n^{2}}}{\sin \phi \tan \phi}} .
$$

The formula given by Drude is

$$
\tan \frac{1}{2} \Delta^{\prime}=\tan \frac{1}{2} \Delta_{0}-\frac{\epsilon}{n} \frac{1}{\sin \phi \tan \phi} .
$$


Surface-Film in Total Reflexion.

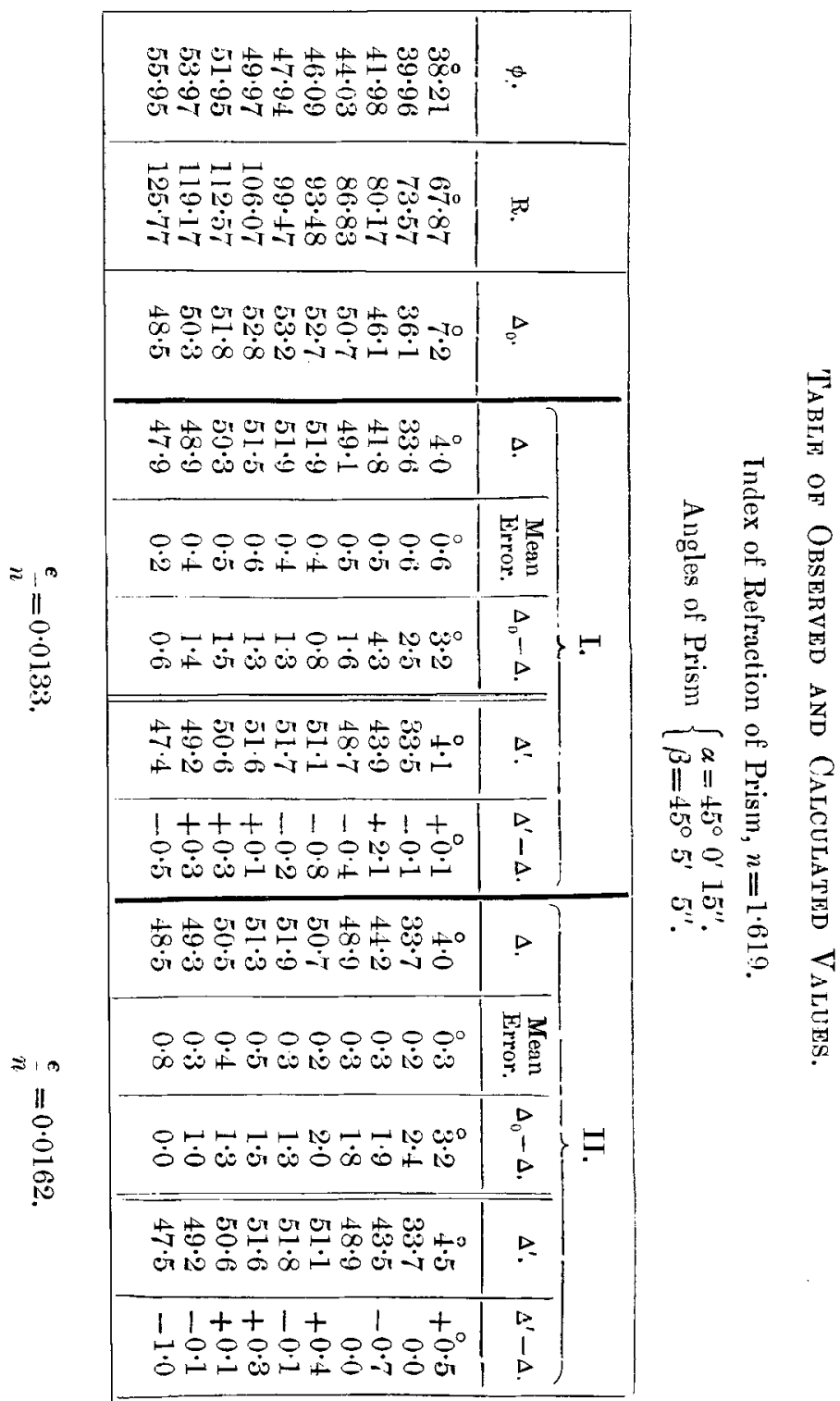


The values of $\phi$ and $\Delta_{0}$ were substituted in the latter formula. Then $\Delta$ was substituted for $\Delta^{\prime}$, and values were obtained for $\epsilon$. The arithmetic mean of these values was then substituted in the second formula, and $\Delta^{\prime}$ calculated for each of the two series of observations.

The effect of the surface-film is very evident.

We have two values for $\frac{\epsilon}{n}, 0 \cdot 0133$ and $0 \cdot() 126$. The mean is

$$
\frac{\epsilon}{u}=0 \cdot 0130
$$

Now $\epsilon$ is defined by the formula

$$
\epsilon=\frac{2 \pi}{\tau \lambda} \frac{n_{1}^{2} n_{0}{ }^{3}}{n_{1}^{2}-n_{0}{ }^{2}} \int_{0}^{\mathrm{T} L} d l\left(\frac{1}{n_{1}{ }^{2}}-\frac{1}{n_{l}^{2}}\right)\left(\frac{1}{n^{2}}-\frac{1}{n_{0}{ }^{2}}\right) n_{l}{ }^{2},
$$

where $L$ is the thickness of the surface-film, $n_{l}$ the index of refraction at the depth $l, n_{0}$ at the depth 0 , and $n_{1}$ at the depth L. $\lambda$ is the wave-length of the light in vacuo.

The fact that $\epsilon$ is positive shows that the mean index of refraction of the film lies between that of the glass and that of the air.

Let us assume that

$$
n_{l}=1 \cdot 309_{5}+0 \cdot 309_{5} \cos \frac{\pi l}{\mathrm{~L}} .
$$

This satisfies the boundary conditions $n_{0}=1 \cdot 619$, and $n_{1}=1$, and goes continuously from the one value to the other. Then $\mathrm{L}$ is found to be $=0.028 \lambda$, where $\lambda$ is the wave-length of the D-lines. In the article of Drude referred to above, a lower limit is found for tho thickness of the surface, from mathematical considerations alone. In the case of glass with the index of refraction $1 \cdot 714$ it is given as $L=0.0103 \lambda$.

The effect of the surface-film is small, and hence difficult to investigate. If the light were several times totally reflected, the effect of the surface-film would be multiplied, and I expected to be able to measure it more accurately. The difficulty was to devise an experimental arrangement which did not call for special apparatus. The diagram shows how this was done.

A pair of Fresnel's rhombs was used. They had been in the laboratory a long time, and had thus developed a good surface-film. The rays of light were totally reflected at $M, N, P$, and $Q$, and thus suffered neither a displacement nor an alteration of direction. The collimator $A$ 
and telescope $B$ remained fixed and opposite to one another during the whole experiment. According to the theory, the phase-differences produced by the surface-film at $R$ and $V$, and at $S$ and $T$ cancel one another. 'The index of refraction of the glass was determined by the method of minimum deviation for each of the four acute angles. The result was $1 \cdot 5195 \pm 2$. The sides were not exactly parallel, the greatest error being as much as $5^{\prime}$. To simplify the calculation, the rhombs were considered perfect, the angles being $54^{\circ} 40^{\prime}$ and $125^{\circ} 20^{\prime}$. To avoid the possibility of error from this approximation, no measurements were made on the first part of the curve, where the phase-difterence increases rapidly with the angle of incidence.

The rhombs were placed on a platform that slid in rails on the spectrometertable. The table could be rotated relatively to the telescopes, and its position read on a scale. The platform was part of the original equipment of the instrument. To determine the angle of incidence and place the rhombs symmetrically, the auxiliary telescope $C$ was used. $\mathrm{C}$ remained always fixed, and the angle between $A$ and $C$ was determined
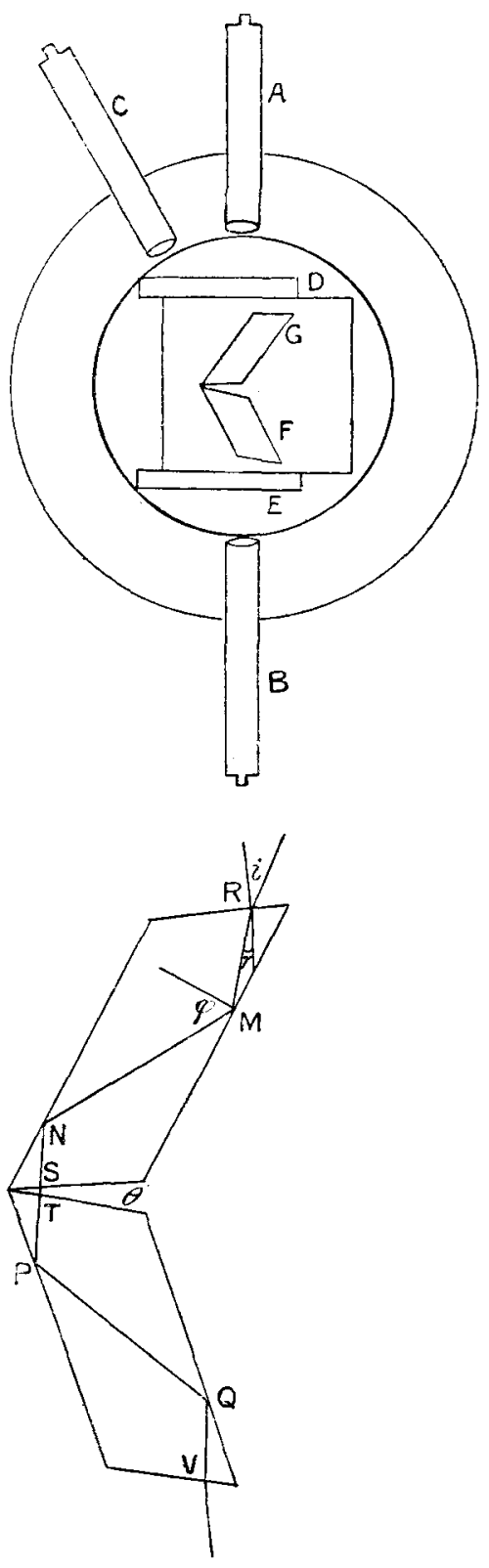

by fitting $C$ with a Gauss ocular, and moving $B$ until the cross-wires in $\mathrm{C}$ coincided with its cross-wires. The angle 
between the two positions of $B$, which could be read on the scale, was the angle between the directions of $\mathrm{A}$ and $\mathrm{C}$. This angle was $41^{\circ} 47^{\prime}$.

Before taking readings of the phase-difference, the table was rotated and the platform adjusted, so that the light from the collimator was reflected on a long side of the first rhomb, making the round opening of the collimator $A$ coincide with the cross-wires of the telescope C. The position of the table was then noted. The same was then done for the second rhomb. If the readings of the scale were in these cases $\alpha$ and $\beta$, and the angle between $A$ and $\mathrm{C} \gamma$, then if the reading on the scale were $\frac{\alpha+\beta}{2}$, the bisector of angle $\theta$ would bisect angle $\gamma$. Consequently, when the rhombs are in the correct position, the scale-reading must be $\frac{\pi+\alpha+\beta-\gamma}{2}$. The table was then placed correctly and the platform displaced until the light from the collimator fell on the most favourable part of the end surfaces.

To find the angle of incidence $\phi$ we proceed as follows. In the position of symmetry $i=\frac{\theta}{2}$,

$$
\sin i=n \sin r=n \sin \left(\phi-54^{\circ} 40^{\prime}\right) \text {. }
$$

And $\theta=\pi-(\beta-\alpha)-2\left(54^{\circ} 40^{\prime}\right)$ : hence $\phi$ can be calculated.

Two series of observations are given in the table. $\Delta, \Delta_{0}$, $\Delta^{\prime}$, and $\phi$ have the same meaning as before.

Observations on Fresnel's Rhombs.

\begin{tabular}{|c|c|c|c|c|c|c|c|c|c|}
\hline \multicolumn{5}{|c|}{ I. } & \multicolumn{5}{|c|}{ II. } \\
\hline$\phi$. & $\Delta$. & $\Delta_{0}$. & $\Delta_{0}-\Delta$ & $\Delta_{0}-\Delta^{\prime}$. & $\phi$. & $\Delta$. & $\Delta_{0}$. & $\Delta_{0}-\Delta$ & $\Delta_{0}-\Delta^{\prime}$ \\
\hline $48^{\circ} \cdot 4$ & $4 \stackrel{\circ}{2} 0$ & $45 \cdot 8$ & 38 & $\begin{array}{l}0 \\
4 \cdot 1\end{array}$ & $51 \cdot 4$ & $4 \ddot{3} \cdot 0$ & $4 \stackrel{0}{6} 6$ & 36 & 37 \\
\hline 51.9 & $43 \cdot 2$ & 46.4 & $3 \cdot 2$ & 3.5 & 52.8 & $42 \cdot 8$ & $46 \cdot 2$ & 34 & $3 \cdot 4$ \\
\hline $54 \cdot 8$ & $42 \cdot 1$ & $45 \cdot 4$ & $3 \cdot 3$ & $3 \cdot 0$ & $54 \cdot 8$ & $42 \div 1$ & $45 \cdot 4$ & $3 \cdot 3$ & $3 \cdot 1$ \\
\hline $56 \cdot 1$ & 425 & 446 & $2 \cdot 1$ & $2 \cdot 8$ & $56 \cdot 5$ & $41 \cdot 4$ & $44 \cdot 2$ & $2 \cdot 8$ & $2 \cdot 8$ \\
\hline $59 \cdot 1$ & $39 \cdot 3$ & $42 \cdot 0$ & $2 \cdot 7$ & $2 \cdot 4$ & $58 \cdot 4$ & $40 \cdot 3$ & $42 \cdot 6$ & $2 \cdot 3$ & 26 \\
\hline $59 \cdot 9$ & 385 & $41 \cdot 2$ & 27 & $2 \cdot 4$ & $59 \cdot 6$ & 393 & $41 \cdot 4$ & $2 \cdot 1$ & 24 \\
\hline $62 \cdot 9$ & 358 & $38 \cdot 0$ & $2 \cdot 2$ & 20 & 62.8 & 356 & 38.1 & 2.6 & $2 \cdot 1$ \\
\hline
\end{tabular}

The accuracy does not seem to be very much greater than in the former case, when the light was only once totally reflected, probably because here the light was not always totally reflected on the same parts of the surface, and the thickness of the surface-film will vary from place to place. 
In both the above cases the film was the natural surfacefilm, and not a homogeneous layer due to polishing, as the glass surfaces had not been polished for a very long time.

An attempt was then made to verify the theory further by depositing very thin artificial surface-films of collodion on the hypotenuse surface of a prism-which had previously shown no trace of such an effect. The artificial film should produce the same effect as the natural films. The films were made after the manner of Wiener, by putting two or three drops of a very dilute solution of collodion in ether and alcohol on the hypotenuse surface of the prism, pressing them down with a similar surface, until they filled all the space between, and then drawing the two surfaces apart. The ether and alcohol then evaporated, leaving a homogeneous skin of collodion.

The prism used was right-angled and isosceles, and the index of refraction for the D-lines was 1:513. The refractive index of collodion is greater than this; and so we had a uniform layer, the index of refraction of which did not lie between that of the glass and that of the air, and which gave an effect in the opposite direction to that produced by the natural film. The film was kept dry during the observations by placing a quantity of calcium chloride on the spectrometer-table. The presence of dampness was easily detected; it lowered the index of refraction of the film to below that of the glass, and we had a deviation from Fresnel's formula in the other direction.

Some trouble was experienced in getting a film of a suitable thickness ; if the film is too thin, the effect is comparable with the errors of measurement, and the formula does not hold when it is too thick. The set of observations given, however, shows the effect of the film very well.

Collodion Film.

Limiting angle of total reflexion, $\phi=41^{\circ} 22^{\prime}$.

\begin{tabular}{|c|c|c|c|c|c|c|c|}
\hline$\phi$. & $\mathbf{R}$ & $\Delta_{0}$ & $\Delta$. & $\begin{array}{l}\text { Mean } \\
\text { Error. }\end{array}$ & $\Delta_{0}-\Delta$ & $\Delta^{\prime}$ & $\Delta^{\prime} \cdots \Delta$ \\
\hline $42^{\circ} 30^{\prime}$ & 8 & $2 \stackrel{0}{25}$ & $2: 2$ & $\stackrel{\circ}{0} \cdot 1$ & 32 & $21 \cdot 6$ & -0.6 \\
\hline 440 & 8658 & $35 \cdot 3$ & 307 & 0.3 & $3 \cdot 6$ & 31.7 & +10 \\
\hline 4530 & 9131 & $40 \div 5$ & $37 \cdot 2$ & $0 \cdot 4$ & $3 \cdot 3$ & $37 \cdot 0$ & $-0 \cdot 2$ \\
\hline 470 & 962 & 435 & $40 \cdot 3$ & $0 \cdot 2$ & 32 & $40 \cdot 2$ & $-0 \cdot 1$ \\
\hline 4830 & 10036 & $45 \cdot 2$ & $4 \cdot 4$ & $0 \cdot 2$ & $2 \cdot 8$ & 422 & $-0 \cdot 2$ \\
\hline 500 & 10510 & $45 \cdot 9$ & 435 & $0 \cdot 1$ & $2 \cdot 4$ & $43 \cdot \mathrm{L}$ & $-0 * t$ \\
\hline 5130 & 10944 & $46 \cdot 1$ & 434 & $0 \cdot 2$ & $2 \cdot 7$ & $43 \cdot 5$ & +0.1 \\
\hline 530 & 11418 & 458 & $43 \cdot 2$ & $0 \cdot 1$ & 26 & $43 \cdot 4$ & $+0 \cdot 2$ \\
\hline 5430 & 11854 & $45 \cdot 2$. & 430 & $0 \cdot 3$ & $2 \cdot 2$ & $42: 9$ & $-0 \cdot 1$ \\
\hline $\begin{array}{ll}560 \\
\end{array}$ & 12328 & $44 \% 3$ & $42 \cdot 1$ & $0 \cdot 3$ & $2 \cdot 1$ & $42 \cdot 2$ & $+0 \cdot 1$ \\
\hline
\end{tabular}

$\epsilon=0.029$.

$\phi, \mathrm{R}, \Delta_{0}, \Delta$, and $\Delta^{\prime}$ have the same meaning as before. 
Attempts were then made to observe the effect of the collodion film in ordinary reflexion and refraction. In the first case the one component was too weak at the polarizing angle to give good results. The phase-difference produced by a surface-film in refraction is given by

$$
\tan \Delta_{d}=-\epsilon \sin \phi \tan \left(\phi-\phi^{\prime}\right) *
$$

where $\phi$ is the angle of incidence and $\phi^{\prime}$ the angle of refraction. The following measurements were obtained :-

\begin{tabular}{|c|c|c|c|}
\hline R. & $\phi$. & $\Delta_{d}$ (air to glass). & $\Delta_{d}$ (glass to air). \\
\hline $154^{\circ}{ }^{\prime}$ & $30^{\circ}$ & $\stackrel{\circ}{0} \cdot 7$ & $-1 \cdot 3$ \\
\hline $154 \quad 12$ & 38 & $0 \cdot 6$ & $-0 \cdot 3$ \\
\hline $153 \quad 22$ & 46 & $0 \cdot 8$ & $-0 \cdot 0$ \\
\hline $51 \quad 32$ & 54 & $0 \cdot 7$ & $-1 \cdot 1$ \\
\hline
\end{tabular}

Owing to some unknown circumstance the measurements from glass to air are not so accurate as those from air to glass. The theoretical value, obtained by using the value of $\epsilon$ already found from observations on total reflexion, is $\pm 0^{\circ} \cdot 6$.

III. Total Reflexion at the Second Surface of a Thin Plane Parallel Plate. By Robert A. Houstocn, M.A., B.Sc., Glasğow University 1851 Exhibition Scholar ${ }^{\circ}$.

THE method used in the experiments described in the preceding paper was also employed to investigate total reflexion at the second surface of a thin plane parallel plate. A prism, the sane as the prism used in the collodion experiment, was coated by Bottger's method with a thin homogeneous layer of silver on its hypotenuse surface. Sodium light, plane-polarized at an angle of $45^{\circ}$ to the plane of incidence, entered the prism, was reflected by the silver layer on the hypotenuse, and the relative phase-difference produced between the components, parallel and perpendicular to the plane of incidence, was measured with the Babinet compensator and analysing nicol. The angle of incidence on the hypotenuse surface was usually such that the light would have been totally reflected, if there had been no silver film there. The silver tilm was then changed into silver iodide, and similar measurements were made.

Theoretically this is the case of the plane parallel plate, the thickness of which is small in comparison with the breadth of the incident light-wave, for the case that the three media

* P. Drude, Wied. Ann. vol. xliii. p. 145 (1891).

$\uparrow$ Communicated by Professor A. Gray, F.R.S. 